\title{
A Placebo-Controlled, Randomized Trial of Enarodustat in Patients with Chronic Kidney Disease Followed by Long-Term Trial
}

\author{
Tadao Akizawa $^{a}$ Masaomi Nangaku ${ }^{b}$ Takuhiro Yamaguchi ${ }^{c}$ Masanobu Arai ${ }^{d}$ \\ Ryosuke Koretomo $^{d}$ Atsushi Matsui $^{d}$ Hideki Hirakata \\ a Division of Nephrology, Department of Medicine, Showa University School of Medicine, Tokyo, Japan; \\ ${ }^{b}$ Division of Nephrology and Endocrinology, The University of Tokyo, Tokyo, Japan; ' Division of Biostatistics,

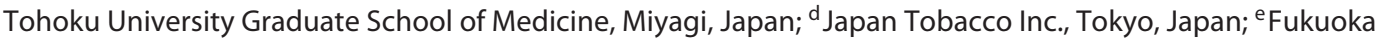 \\ Renal Clinic, Fukuoka, Japan
}

\section{Keywords}

Enarodustat - Anemia in chronic kidney disease .

Hypoxia-inducible factor prolyl hydroxylase inhibitor .

Hepcidin · Randomized trial

\begin{abstract}
Background: Enarodustat (JTZ-951) is an oral hypoxia-inducible factor prolyl hydroxylase inhibitor that mimics adaptive responses to hypoxic conditions and may provide a new therapeutic approach for managing anemia in patients with chronic kidney disease (CKD). We evaluated the efficacy, safety, and maintenance dose of enarodustat in anemic patients with CKD not on dialysis. Methods: Erythropoiesisstimulating agent (ESA) naïve patients (correction group) and patients on a stable dose of ESA (conversion group) were randomized to receive 2,4 , or $6 \mathrm{mg}$ of enarodustat or placebo once daily for 6 weeks in a double-blind manner ( $\mathrm{Pe}$ riod 1) followed by 24 weeks of open enarodustat treatment to maintain their hemoglobin $(\mathrm{Hb})$ levels within a target range of $10.0-12.0 \mathrm{~g} / \mathrm{dL}$ in reference to a dose adjustment algorithm (Period 2). Results: In the correction group, $\mathrm{Hb}$ level increase rate per week increased in a dose-response manner. The proportion of subjects in the conversion group who maintained $\mathrm{Hb}$ levels within $\pm 1.0 \mathrm{~g} / \mathrm{dL}$ of baseline did not
\end{abstract}

differ between each enarodustat arm and placebo arm during Period 1 . Over $70 \%$ of subjects in both groups maintained $\mathrm{Hb}$ levels within the target range at the end of treatment in Period 2. The mean prescribed doses were 3.58 and $3.74 \mathrm{mg} /$ day in the correction group and the conversion group, respectively. Enarodustat was associated with decreases in hepcidin and ferritin and increased total ironbinding capacity and was generally well tolerated. Conclusions: Enarodustat corrects and maintains $\mathrm{Hb}$ levels in anemic patients with CKD not on dialysis.

C 2019 The Author(s)
Published by S. Karger AG, Basel

\section{Introduction}

Renal anemia, a common complication of chronic kidney disease (CKD), mainly results from reduced erythropoietin (EPO) production by the kidney [1] and other factors including altered iron metabolism [2] and shortened erythrocyte lifespan [3]. Renal anemia is an independent risk factor for CKD progression and cardiovascular disease [4-6] and is therefore an important therapeutic target in patients with CKD.

\begin{tabular}{ll}
\hline KARGER & $\begin{array}{l}\text { (c) } 2019 \text { The Author(s) } \\
\text { Published by S. Karger AG, Basel }\end{array}$ \\
E-Mail karger@karger.com & This article is licensed under the Creative Commons Attribution- \\
www.karger.com/ajn & $\begin{array}{l}\text { NonCommercial-NoDerivatives 4.0 International License (CC BY- } \\
\text { NC-ND) (http://www.karger.com/Services/OpenAccessLicense). } \\
\text { Usage and distribution for commercial purposes as well as any dis- } \\
\text { tribution of modified material requires written permission. }\end{array}$
\end{tabular}


Erythropoiesis-stimulating agents (ESAs) are a standard therapy for renal anemia; however, administration of ESAs with a high hemoglobin $(\mathrm{Hb})$ target or in high dosage may be associated with an increased risk of cardiovascular events and mortality [7-10]. Moreover, injection of ESAs is painful, and administration of ESAs may contribute to increased risk of infection, especially in patients with CKD not on hemodialysis.

EPO production is regulated by hypoxia-inducible factor (HIF) $[11,12]$. The activity of the HIF-a subunit is regulated by prolyl hydroxylase $(\mathrm{PH})$ domain-containing protein, the activity of which is suppressed under hypoxic conditions, resulting in stabilized HIF- $\alpha[13,14]$.

HIF regulates the expression of genes encoding hepcidin, which is involved in iron absorption and homeostasis. Regulation of hepatic hepcidin mRNA expression has been reported in transgenic mice in which HIF has been stabilized [15].

The HIF-PH inhibitor enarodustat (JTZ-951) is an orally available agent for treatment of anemia by stabilizing HIF- $\alpha$ in the kidney and liver and increasing endogenous EPO production. Moreover, it is expected that enarodustat stimulates erythropoiesis by improving iron absorption and utilization by stabilizing HIF- $\alpha$. A Phase 2a clinical study in Japanese patients with CKD not on dialysis showed that taking enarodustat for 6 weeks in escalating doses of 1-5 mg increased $\mathrm{Hb}$ levels from baseline without safety concerns [16]. The objective of the current Phase $2 \mathrm{~b}$ study was to evaluate the efficacy and safety of taking enarodustat in doses of $2-8 \mathrm{mg}$ for up to 30 weeks as both initial and maintenance therapy for anemia in Japanese patients with CKD not on dialysis.

\section{Materials and Methods}

\section{Study Design}

This study was conducted at 54 institutions from May 2015 to September 2016 and consisted of a 4-week screening (Scr) period, 30 -week treatment period, and 2 -week follow-up period. The study design is a randomized, double-blind, placebo-controlled, parallel-arm comparison study to assess short-term efficacy and safety during the first 6 weeks of the treatment period (Period 1); and an open-label, uncontrolled, intraindividual dose-adjustment study to assess long-term safety and maintenance dose of enarodustat in the subsequent 24 weeks (Period 2). The study cohort consisted of anemic patients with CKD not on dialysis. These patients were divided into 2 groups: a correction group, comprising ESA-naïve patients (ESA not received for $\geq 12$ weeks before Scr Visit 1); and a conversion group, comprising ESA-treated patients (stable ESA treatment for $\geq 8$ weeks before Scr Visit 1).

For Period 1, subjects were randomly assigned to enarodustat 2,4 , or $6 \mathrm{mg}$, or placebo once daily for 6 weeks in a ratio of 1:1:1:
1. In Period 2, enarodustat was taken once daily for 24 weeks at a starting dose of $2 \mathrm{mg}$ ( $\mathrm{Hb}$ level at Week 6 of $>12.0$ and $<13.0 \mathrm{~g} / \mathrm{dL}$ ) or $4 \mathrm{mg}$ ( $\mathrm{Hb}$ level at Week 6 of $\geq 8.0$ and $\leq 12.0 \mathrm{~g} / \mathrm{dL}$ ), with subsequent doses adjusted every 4 weeks within $2-8 \mathrm{mg}$ to maintain $\mathrm{Hb}$ levels within the target range (10.0-12.0 g/dL). Treatment was suspended if $\mathrm{Hb}$ reached $\geq 13.0 \mathrm{~g} / \mathrm{dL}$.

The study was registered with the Japan Pharmaceutical International Center (JapicCTI-152881) and was conducted in compliance with the ethical principles of the Declaration of Helsinki, the protocol, and the Guidelines for Good Clinical Practice of the Japanese Ministerial Ordinance, and was approved by the Institutional Review Board of each participating study site. All patients provided written informed consent prior to participation.

\section{Subjects and Treatment}

Eligibility criteria for Period 1 were aged $\geq 20$ years, CKD not on dialysis (estimated glomerular filtration rate $<60 \mathrm{~mL} /$ $\mathrm{min} / 1.73 \mathrm{~m}^{2}$ ), mean $\mathrm{Hb}$ level at Scr Visit 1 and 2 weeks later $(\mathrm{Scr}$ Visit 2) of 8.0-10.5 g/dL for the correction group, and 9.5-12.0 g/ $\mathrm{dL}$ for the conversion group. Patients who had received an erythrocyte transfusion or undergone surgery involving massive blood loss within 12 weeks before Scr Visit 1 and those suspected of having anemia caused by noninfectious chronic inflammatory disease were excluded. Further details of eligibility criteria are summarized in online supplementary Materials (for all online suppl. material, see www. karger.com/doi/10.1159/000496929).

Subjects who completed Period 1 entered Period 2 if their $\mathrm{Hb}$ level at Week 6 was $\geq 8.0$ and $<13.0 \mathrm{~g} / \mathrm{dL}$.

During the Scr period and Period 1, intravenous iron preparations were prohibited and oral iron preparations were permitted only if they had been used before Scr Visit 1; however, a change in dose regimen was prohibited. During Period 2, iron replacement therapy was permitted as considered necessary.

\section{Assessments}

The primary endpoint in Period 1 was $\mathrm{Hb}$ level increase rate per week for the correction group and proportion of subjects who maintained a change from baseline $\mathrm{Hb}$ level within $\pm 1.0 \mathrm{~g} / \mathrm{dL}$ at the evaluation point for the conversion group. Baseline was defined as the value at Week 0 for the correction group and the mean value at Scr Visit 1, Scr Visit 2, and Week 0 for the conversion group. $\mathrm{Hb}$ level at the evaluation point was the average of the last $2 \mathrm{Hb}$ levels for the conversion group (i.e., end of Period 1 treatment and the previous time point).

Efficacy assessments in Period 2 included the time-course of $\mathrm{Hb}$ levels, proportion of subjects who maintained $\mathrm{Hb}$ levels within the target range ( $\geq 10.0$ and $\leq 12.0 \mathrm{~g} / \mathrm{dL})$, mean prescribed dose, and number of dose adjustments. No primary endpoint was set for Period 2.

Safety assessments included adverse events (AEs) occurring after the start of treatment, laboratory findings, vital signs, standard 12-lead electrocardiogram, chest X-ray, and fundoscopy.

In addition, iron-related parameters were assessed.

\section{Statistical Analysis}

All analyses were performed using SAS version 9.3 or higher (SAS Institute, Cary, NC, USA).

It was assumed on the basis of previous clinical studies [16] that the mean $\mathrm{Hb}$ level increase rate would be $0,0.1,0.25$, and $0.4 \mathrm{~g} / \mathrm{dL} /$ week in the placebo, 2, 4, and $6 \mathrm{mg}$ arms, respectively, with a SD 
of $0.35 \mathrm{~g} / \mathrm{dL} /$ week. Based on these assumptions, the number of subjects necessary for one-way analysis of variance with $2.5 \%$ onesided significance level, $90 \%$ detection power, and contrast of monotonic increase $(-3,-1,1,3)$ was determined as 15 per arm, resulting in a sample size of 20 subjects per arm in the correction group, allowing for withdrawals. Again on the basis of previous clinical studies [16], it was assumed that $10 \%$ of subjects in the placebo arm and $70 \%$ of those in the enarodustat arm would maintain an $\mathrm{Hb}$ level within the range of baseline $\pm 1.0 \mathrm{~g} / \mathrm{dL}$. Based on these assumptions, the number of subjects necessary for Fisher's exact test with $2.5 \%$ one-sided significance level and the $90 \%$ power of detection was determined as 16 per arm, resulting in a sample size of 20 subjects per arm in the conversion group.

The efficacy full analysis set (FAS) in Period 1 comprised subjects who received the study drug and were assessed for efficacy at least once for the correction group and at least twice for the conversion group. The FAS in Period 2 comprised subjects assessed for efficacy at least once in Period 2. The safety population consisted of subjects who received the study drug and were assessed for safety at least once.

The primary endpoint in the correction group was assessed by a trend test for monotonic increase (contrast: $-3,-1,1,3$ ) using a mixed effect model with treatment arm and treatment arm-bytime interaction as fixed effects and intercept and time as random effects (significance level: 2.5\%, one-sided). For secondary analysis of the primary endpoint, the least squares mean was determined for each treatment arm, and the least squares mean of inter-arm difference from the placebo arm and its 95\% CI and $p$ value were determined. Adjustment for multiplicity was not performed. The primary endpoint in the conversion group was assessed by comparing the placebo arm with each enarodustat arm using Fisher's exact test (significance level: $2.5 \%$, one-sided). Adjustment for multiplicity was performed by permutation testing. For the conversion group, the proportion of subjects with an $\mathrm{Hb}$ level at the evaluation point within $\pm 1.0 \mathrm{~g} / \mathrm{dL}$ of baseline was analyzed as post hoc analysis using Fisher's exact test to demonstrate differences in proportions of subjects in the enarodustat 2 and $4 \mathrm{mg}$ arms than in the other 2 arms.

Iron-related parameters in each group were assessed in the safety population only. Post hoc analysis was performed to compare changes in each iron-related parameter from Week 0 to Week 6 between the placebo and each enarodustat arm using a Wilcoxon rank sum test (significance level: 5\%, 2-sided). Given that this was an exploratory investigation, adjustment for multiplicity was not performed.

AEs reported between the start of study treatment and completion were coded using MedDRA/J version 18.0 and tabulated.

\section{Results}

\section{Study Subjects' Characteristics}

Ninety-four subjects in the correction group and 107 in the conversion group were randomized to receive placebo or one of the 3 enarodustat arms. One subject in the conversion group was withdrawn after randomization but before the initiation of study treatment and therefore excluded from the FAS and safety population, whereas 3 more in the conversion group were assessed for efficacy less than twice and therefore excluded from the FAS. Thus, 94 subjects in the correction group were included in the FAS and safety population and 103 and 106 in the conversion group in the FAS and safety population, respectively. Period 1 was completed by 77 subjects in the correction group and 97 in the conversion group.

Period 2 included 77 subjects in the correction group and 90 in the conversion group; 66 and 77 subjects, respectively, completed Period 2.

Relevant subject characteristics according to group are shown in Table 1 and a subject disposition in online supplementary Figure S1.

\section{Primary Endpoint (Correction Group, Period 1)}

Mean $\mathrm{Hb}$ level at baseline was $9.81 \pm 0.63 \mathrm{~g} / \mathrm{dL}$ in the placebo arm, $9.69 \pm 0.76 \mathrm{~g} / \mathrm{dL}$ in the enarodustat $2 \mathrm{mg}$ arm, $9.77 \pm 0.74 \mathrm{~g} / \mathrm{dL}$ in the $4 \mathrm{mg}$ arm, and $9.32 \pm 0.67 \mathrm{~g} /$ $\mathrm{dL}$ in the $6 \mathrm{mg}$ arm. Hb level increase rate per week (least squares mean $\pm \mathrm{SE}$ ) estimated using the mixed effect model was $-0.023 \pm 0.034 \mathrm{~g} / \mathrm{dL} /$ week in the placebo arm, $0.137 \pm 0.034 \mathrm{~g} / \mathrm{dL} /$ week in the $2 \mathrm{mg}$ arm, $0.193 \pm 0.034$ $\mathrm{g} / \mathrm{dL} /$ week in the $4 \mathrm{mg}$ arm, and $0.440 \pm 0.037 \mathrm{~g} / \mathrm{dL} /$ week in the $6 \mathrm{mg}$ arm, showing a dose-related response to enarodustat by trend testing (monotonic increase; $p<$ 0.0001).

The inter-arm difference in the least squares mean in rate of increase in $\mathrm{Hb}$ level estimated using the mixed effect model was $0.160 \mathrm{~g} / \mathrm{dL} /$ week in the $2 \mathrm{mg}$ arm, $0.216 \mathrm{~g} /$ $\mathrm{dL} /$ week in the $4 \mathrm{mg}$ arm, and $0.464 \mathrm{~g} / \mathrm{dL} /$ week in the $6 \mathrm{mg}$ arm, showing a significantly higher rate of increase in Hb level in all enarodustat than placebo arm $(2 \mathrm{mg}, p=$ 0.0015; $4 \mathrm{mg}$ and $6 \mathrm{mg}, p<0.0001)$.

\section{Primary Endpoint (Conversion Group, Period 1)}

The mean $\mathrm{Hb}$ level at baseline was $10.73 \pm 0.61 \mathrm{~g} / \mathrm{dL}$ in the placebo arm, $10.89 \pm 0.57 \mathrm{~g} / \mathrm{dL}$ in the enarodustat 2 $\mathrm{mg}$ arm, $10.73 \pm 0.71 \mathrm{~g} / \mathrm{dL}$ in the $4 \mathrm{mg}$ arm, and $10.53 \pm$ $0.63 \mathrm{~g} / \mathrm{dL}$ in the $6 \mathrm{mg}$ arm. At the evaluation point, $54.2 \%$ of subjects in the placebo arm, $80.8 \%$ in the enarodustat $2 \mathrm{mg}$ arm, $70.4 \%$ in the $4 \mathrm{mg}$ arm, and $50.0 \%$ in the $6 \mathrm{mg}$ arm maintained $\mathrm{Hb}$ levels within $\pm 1.0 \mathrm{~g} / \mathrm{dL}$ of baseline; these differences were not significant. Post hoc analysis using Fisher's exact test indicated a statistically significant difference in the proportion of subjects with an $\mathrm{Hb}$ level within the baseline value $\pm 1.0 \mathrm{~g} / \mathrm{dL}$ at the evaluation point between the enarodustat $2 \mathrm{mg}$ and $4 \mathrm{mg}$ arms and the other 2 arms $(p=0.0112)$. 
Table 1. Subject characteristics

a. Correction group

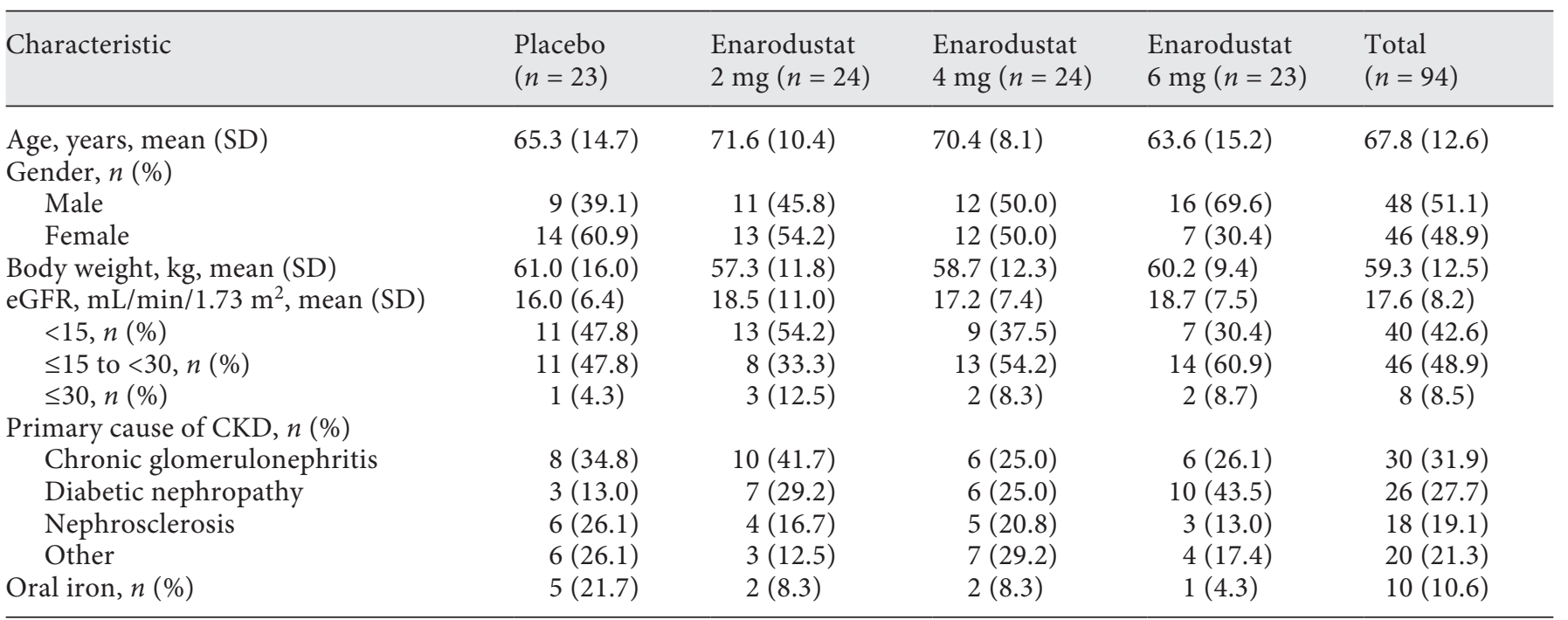

b. Conversion group

\begin{tabular}{|c|c|c|c|c|c|}
\hline Characteristic & $\begin{array}{l}\text { Placebo } \\
(n=24)\end{array}$ & $\begin{array}{l}\text { Enarodustat } \\
2 \mathrm{mg}(n=26)\end{array}$ & $\begin{array}{l}\text { Enarodustat } \\
4 \mathrm{mg}(n=27)\end{array}$ & $\begin{array}{l}\text { Enarodustat } \\
6 \mathrm{mg}(n=26)\end{array}$ & $\begin{array}{l}\text { Total } \\
(n=103)\end{array}$ \\
\hline Age, years, mean (SD) & $66.2(11.9)$ & $67.8(9.3)$ & $67.5(11.8)$ & $68.8(11.2)$ & $67.6(11.0)$ \\
\hline \multicolumn{6}{|l|}{ Gender, $n(\%)$} \\
\hline Male & $13(54.2)$ & $15(57.7)$ & $14(51.9)$ & $12(46.2)$ & $54(52.4)$ \\
\hline Female & $11(45.8)$ & $11(42.3)$ & $13(48.1)$ & $14(53.8)$ & $49(47.6)$ \\
\hline Body weight, kg, mean (SD) & $57.1(11.2)$ & $62.8(10.5)$ & $57.0(11.6)$ & $53.0(10.2)$ & $57.5(11.3)$ \\
\hline $\mathrm{eGFR}, \mathrm{mL} / \mathrm{min} / 1.73 \mathrm{~m}^{2}$, mean $(\mathrm{SD})$ & $16.4(8.1)$ & $14.9(5.8)$ & $14.3(5.9)$ & $17.2(7.3)$ & $15.7(6.8)$ \\
\hline$<15, n(\%)$ & $15(62.5)$ & $13(50.0)$ & $17(63.0)$ & $13(50.0)$ & $58(56.3)$ \\
\hline$\leq 15$ to $<30, n(\%)$ & $5(20.8)$ & $13(50.0)$ & $9(33.3)$ & $10(38.5)$ & $37(35.9)$ \\
\hline$\leq 30, n(\%)$ & $4(16.7)$ & $0(0.0)$ & $1(3.7)$ & $3(11.5)$ & $8(7.8)$ \\
\hline \multicolumn{6}{|l|}{ Primary disease of CKD, $n(\%)$} \\
\hline Chronic glomerulonephritis & $11(45.8)$ & $10(38.5)$ & $4(14.8)$ & $11(42.3)$ & $36(35.0)$ \\
\hline Diabetic nephropathy & $5(20.8)$ & $6(23.1)$ & $7(25.9)$ & $3(11.5)$ & $21(20.4)$ \\
\hline Nephrosclerosis & $2(8.3)$ & $7(26.9)$ & $11(40.7)$ & $6(23.1)$ & $26(25.2)$ \\
\hline Other & $6(25.0)$ & $3(11.5)$ & $5(18.5)$ & $6(23.1)$ & $20(19.4)$ \\
\hline \multicolumn{6}{|l|}{ Prior ESA, $n(\%)$} \\
\hline rHuEPO & $1(4.2)$ & $2(7.7)$ & $2(7.4)$ & $3(11.5)$ & $8(7.8)$ \\
\hline Darbepoetin alfa & $12(50.0)$ & $9(34.6)$ & $14(51.9)$ & $11(42.3)$ & $46(44.7)$ \\
\hline Epoetin beta pegol & $11(45.8)$ & $15(57.7)$ & $11(40.7)$ & $12(46.2)$ & $49(47.6)$ \\
\hline \multicolumn{6}{|l|}{ Prior ESA dose per week } \\
\hline rHuEPO, IU, mean & 3,000 & 1,500 & 3,000 & 2,500 & $2,437.5(776.3)$ \\
\hline Darbepoetin alfa, $\mu \mathrm{g}$, mean (SD) & $13.3(6.3)$ & $13.1(7.9)$ & $13.9(6.3)$ & $16.8(11.2)$ & $14.3(7.9)$ \\
\hline Epoetin beta pegol, $\mu \mathrm{g}$, mean (SD) & $68.2(47.6)$ & $71.7(49.9)$ & $56.8(31.8)$ & $58.3(22.2)$ & $64.3(39.5)$ \\
\hline Oral iron, $n(\%)$ & $3(12.5)$ & $3(11.5)$ & $2(7.4)$ & $4(15.4)$ & $12(11.7)$ \\
\hline
\end{tabular}

Body weight and eGFR on Scr Visit 1 are shown. The number of patients taking oral iron at Scr Visit 1 is shown.

CKD, chronic kidney disease; eGFR, estimated glomerular filtration rate; ESA, erythropoiesis-stimulating agent; rHuEPO, recombinant human erythropoietin; Scr, screening. 
Fig. 1. Changes in hemoglobin levels over time. (a) Correction group; (b) conversion group. Each point indicates mean hemoglobin level in each treatment arm on each observation day; bars indicate SD. Ext, extention; F-up, follow-up.

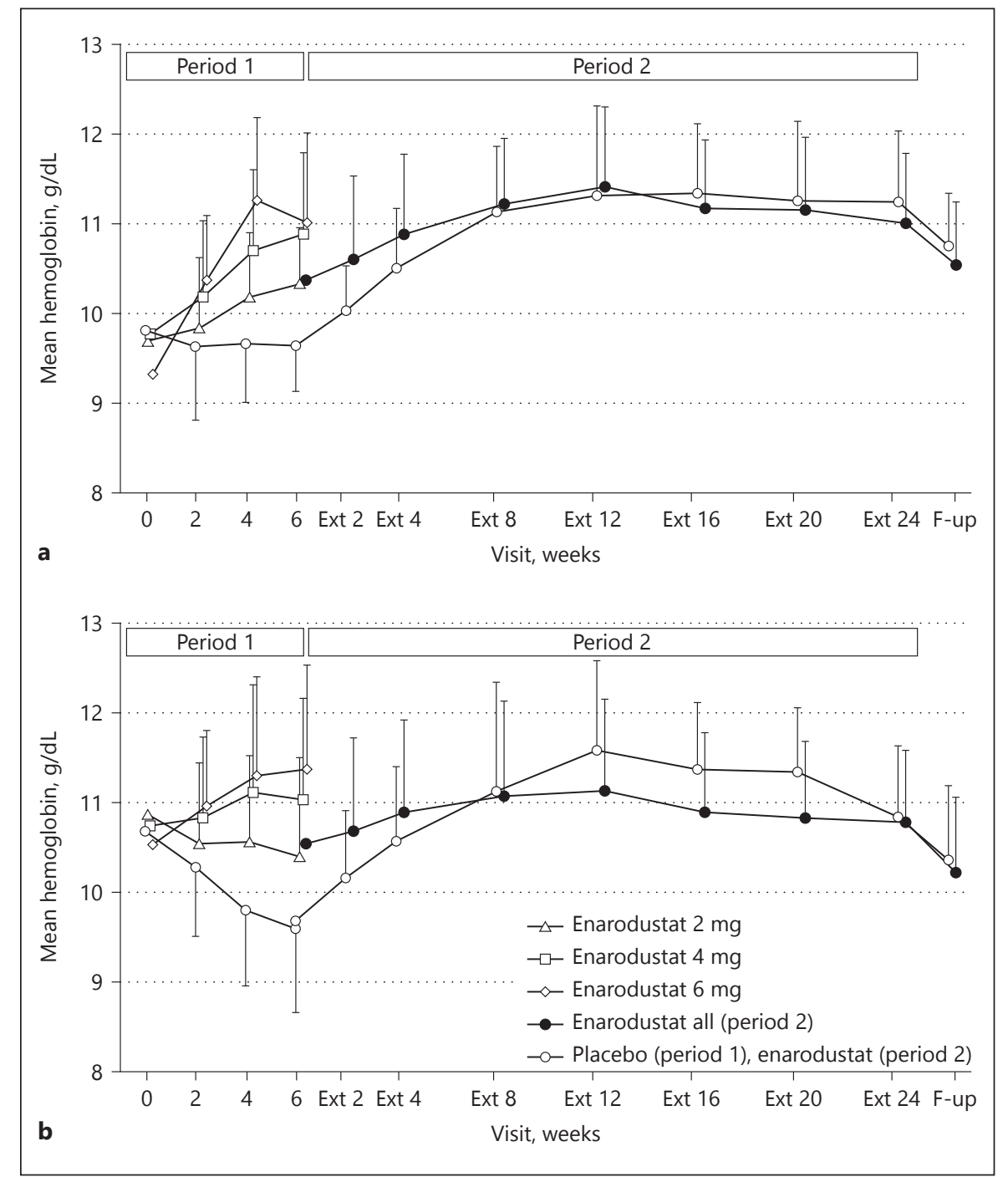

\section{Maintenance of $\mathrm{Hb}$ (Period 2)}

In both groups, 24-week enarodustat treatment with dose adjustment maintained $\mathrm{Hb}$ levels within the target range of $10.0-12.0 \mathrm{~g} / \mathrm{dL}$ (Fig. 1). At the end of treatment, $71.4 \%$ of subjects in the correction group and $78.9 \%$ in the conversion group maintained $\mathrm{Hb}$ level within the target range. For the subjects assigned to the placebo arm in Period 1 and moved to Period 2, the target range maintenance rate was $39.1 \%$ in the correction group and $43.5 \%$ in the conversion group at Week 6. When placebo was switched to enarodustat, the rate increased to 77.3 and $73.9 \%$ in each group at Extension (Ext) Week 4 (4 weeks after the start of Period 2 treatment) and remained at $\geq 70 \%$ thereafter.

The mean prescribed dose in Period 2 was $3.58 \mathrm{mg} /$ day in the correction group and $3.74 \mathrm{mg} /$ day in the conver- sion group. Drug compliance in Period 2 was $98.8 \%$ in the correction group and $98.6 \%$ in the conversion group. No dose adjustment was required in $37.7 \%$ of subjects in the correction group and $25.6 \%$ in the conversion group during Period 2. Additionally, more than $70 \%$ of subjects in both groups required fewer than 2 dose adjustments.

\section{Iron-Related Parameters}

The time courses of change in iron-related parameters in Period 1 are shown in Figure 2. Iron-related parameters at Week 0 (baseline) are shown in online supplementary Table S1.

Hepcidin and ferritin decreased and total iron-binding capacity (TIBC) increased in the enarodustat arms. Post hoc analysis showed that the changes in hepcidin, ferritin, and TIBC at Week 6 were significantly greater in 


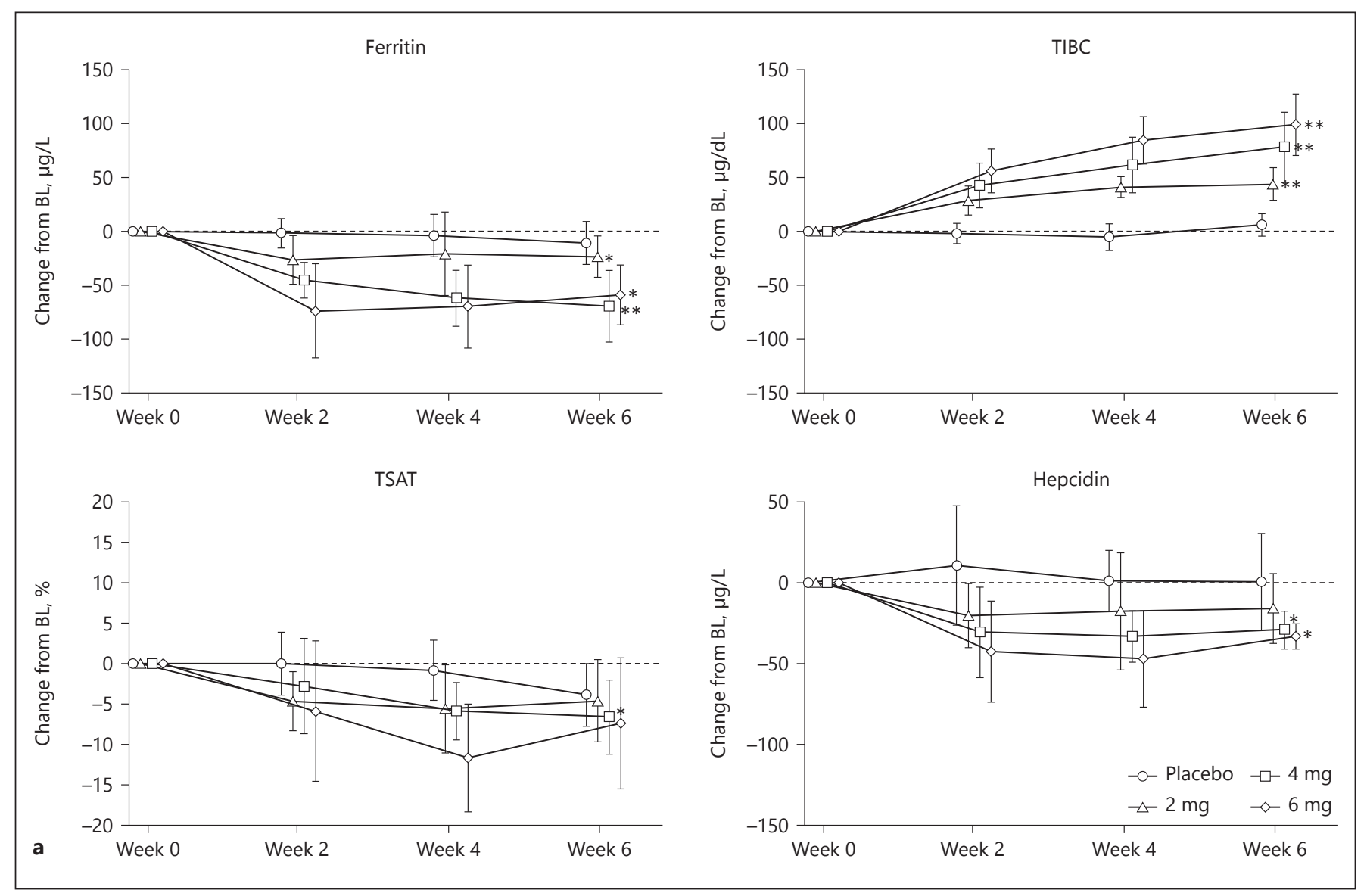

Fig. 2. Changes in iron-related parameters (Period 1). (a) Correction group, Period 1; (b) conversion group, Period 1. Each point indicates the median value on each observation day in each treatment arm; bars indicate quartile deviations. Post hoc analysis was performed only for changes in Week 6 , which were compared be-

tween the placebo arm and each enarodustat arm using the Wilcoxon rank sum test (significance level: 5\%, 2-sided). Adjustment for multiplicity was not performed. ${ }^{*} p<0.05,{ }^{* *} p<0.0001$. BL, baseline; TIBC, total iron-binding capacity; TSAT, transferrin saturation.

(For figure $2 b$ see next page.)

the enarodustat 4 and $6 \mathrm{mg}$ arms than in the placebo arm. These parameters remained stable in Period 2.

No subjects received intravenous iron preparations in Period 2; however, 19 of $77(24.7 \%)$ in the correction group and 15 of $90(16.7 \%)$ in the conversion group received oral iron preparations in Period 2.

\section{Safety}

No deaths occurred during this study. In Period 1, 33 of $71(46.5 \%)$ subjects receiving enarodustat and 8 of 23 (34.8\%) receiving placebo in the correction group, and 21 of $80(26.3 \%)$ subjects receiving enarodustat and 5 of 26 (19.2\%) receiving placebo in the conversion group developed AEs. Serious AEs (SAEs) in Period 1 were reported in one subject receiving enarodustat $4 \mathrm{mg}$ (peripheral arterial occlusive disease) in the correction group, and one receiving enarodustat $2 \mathrm{mg}$ (bronchitis bacterial), 2 receiving $4 \mathrm{mg}$ (one retinal detachment, one renal cyst infection), and one receiving placebo (sinus arrest, renal failure, and cardiac failure) in the conversion group. All SAEs in Period 1 were considered unrelated to the study drug. Increases in enarodustat dose were not associated with increases in SAEs.

AEs reported in at least $5 \%$ of subjects in any group after taking enarodustat, and all SAEs reported after taking enarodustat are summarized in Table 2. SAEs were reported in 5 subjects in the correction group and 12 in the conversion group after taking enarodustat. Of all SAEs, causal relationships with the study drug could not be excluded for bone cancer or deep vein thrombosis.

There were no clinically significant changes in laboratory findings, vascular endothelial growth factor (VEGF), 


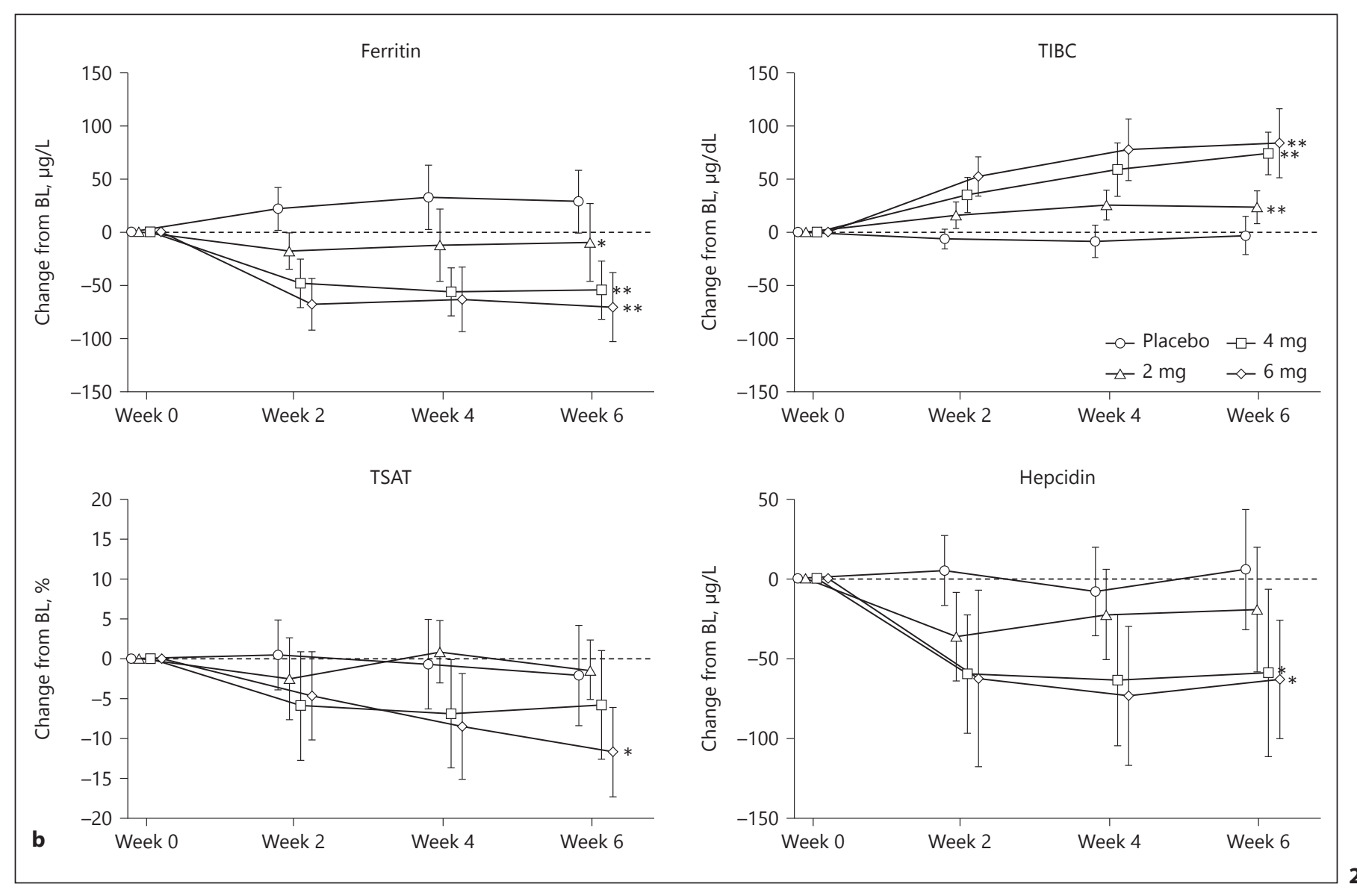

EPO, vital signs, standard 12-lead electrocardiogram, chest X-ray, fundoscopy, estimated glomerular filtration rate, or urine protein.

\section{Discussion/Conclusion}

In Period 1, enarodustat increased Hb levels in a doseresponse manner in the correction group. Out of 23 subjects in the enarodustat $6 \mathrm{mg}$ arm in the correction group, 11 subjects discontinued the study due to the $\mathrm{Hb}$-related discontinuation criterion (i.e., $\mathrm{Hb}$ increases $>2.0 \mathrm{~g} / \mathrm{dL}$ in 4 weeks; online suppl. Fig. S1a), which suggested that the recommended initial dose is considered to be less than $6 \mathrm{mg}$ in CKD patients who are not on ESAs. In the conversion group, the proportion of subjects with an $\mathrm{Hb} \mathrm{lev-}$ el within baseline $\pm 1.0 \mathrm{~g} / \mathrm{dL}$ after switching from an ESA in each enarodustat arm did not differ significantly from that in the placebo arm. As shown in the Figure 1b, the $\mathrm{Hb}$ levels at Week 6 increased with dose, resulting in the primary endpoint in the enarodustat $6 \mathrm{mg}$ arm tended to

be lower than those in other 2 enarodustat arms. However, post hoc trend testing of maintenance of $\mathrm{Hb}$ level showed a statistically significant difference between the 2 and $4 \mathrm{mg}$ arms and the other 2 arms. Thus, a relationship between enarodustat dose and $\mathrm{Hb}$ response was found. In Period 2, Hb levels were maintained within the target range at each observation after Ext Week 4 in $\geq 70 \%$ of subjects by adjusting the dose of enarodustat in the range of $2-8 \mathrm{mg}$ on the basis of $\mathrm{Hb}$ level. Furthermore, more than $70 \%$ of subjects required one or no dose adjustment during Period 2.

While the efficacy and safety of ESAs treating patients with anemia associated with CKD have been established, enarodustat has unique characteristics in that it can be stored at room temperature and taken orally, avoiding the pain associated with injections in general.

$\mathrm{Hb}$ level at the Ext follow-up visit (2 weeks after Ext Week 24) was lower than in Ext Week 24, suggesting that the effect of enarodustat on $\mathrm{Hb}$ is attenuated within 2 weeks and that $\mathrm{Hb}$ levels above the upper limit of the target control range decrease rapidly after drug withdrawal. 
Table 2. AEs reported in $5 \%$ or more subjects and any SAEs

\begin{tabular}{|c|c|c|c|}
\hline & \multicolumn{3}{|c|}{ Enarodustat treatment } \\
\hline & $\begin{array}{l}\text { correction group } \\
(n=94)\end{array}$ & $\begin{array}{l}\text { conversion group } \\
(n=103)\end{array}$ & total $(n=197)$ \\
\hline \multicolumn{4}{|l|}{ AEs ( $\geq 5 \%$ subjects), $n(\%)$} \\
\hline Any AEs & $71(75.5)$ & $78(75.7)$ & $149(75.6)$ \\
\hline Nasopharyngitis & $26(27.7)$ & $25(24.3)$ & $51(25.9)$ \\
\hline Hypertension & $8(8.5)$ & $5(4.9)$ & $13(6.6)$ \\
\hline Hyperkalemia & $3(3.2)$ & $6(5.8)$ & $9(4.6)$ \\
\hline Constipation & $5(5.3)$ & $3(2.9)$ & $8(4.1)$ \\
\hline \multicolumn{4}{|l|}{ Serious AEs, $n(\%)$} \\
\hline Peripheral arterial occlusive disease & $1(1.1)^{\mathrm{a}}$ & $0(0.0)$ & $1(0.5)$ \\
\hline Inguinal hernia & $1(1.1)$ & $0(0.0)$ & $1(0.5)$ \\
\hline Aortic dissection & $1(1.1)^{b}$ & $0(0.0)$ & $1(0.5)$ \\
\hline Large intestine polyp & $1(1.1)$ & $0(0.0)$ & $1(0.5)$ \\
\hline Colon cancer & $1(1.1)$ & $0(0.0)$ & $1(0.5)$ \\
\hline Cardiac failure congestive & $1(1.1)^{\mathrm{b}}$ & $0(0.0)$ & $1(0.5)$ \\
\hline Edema peripheral & $0(0.0)$ & $1(1.0)$ & $1(0.5)$ \\
\hline Enterocolitis & $0(0.0)$ & $1(1.0)$ & $1(0.5)$ \\
\hline Loss of consciousness & $0(0.0)$ & $1(1.0)$ & $1(0.5)$ \\
\hline Retinal detachment & $0(0.0)$ & $1(1.0)^{\mathrm{a}}$ & $1(0.5)$ \\
\hline Renal cyst infection & $0(0.0)$ & $1(1.0)^{\mathrm{a}}$ & $1(0.5)$ \\
\hline Meniscus injury & $0(0.0)$ & $1(1.0)$ & $1(0.5)$ \\
\hline Bone cancer & $0(0.0)$ & $1(1.0)^{\mathrm{c}}$ & $1(0.5)$ \\
\hline Bronchitis bacterial & $0(0.0)$ & $1(1.0)^{\mathrm{a}, \mathrm{d}}$ & $1(0.5)$ \\
\hline Pancreatitis acute & $0(0.0)$ & $1(1.0)$ & $1(0.5)$ \\
\hline Pyelonephritis & $0(0.0)$ & $1(1.0)$ & $1(0.5)$ \\
\hline Sepsis & $0(0.0)$ & $1(1.0)$ & $1(0.5)$ \\
\hline Postrenal failure & $0(0.0)$ & $1(1.0)$ & $1(0.5)$ \\
\hline Atrioventricular block complete & $0(0.0)$ & $1(1.0)$ & $1(0.5)$ \\
\hline Sarcoidosis & $0(0.0)$ & $1(1.0)$ & $1(0.5)$ \\
\hline Deep vein thrombosis & $0(0.0)$ & $1(1.0)^{b, c}$ & $1(0.5)$ \\
\hline $\begin{array}{l}\text { a Serious AE reported in Period } 1 . \\
\text { b Serious AE requiring discontinuati } \\
{ }^{c} \text { Serious AEs judged by the principa } \\
\text { d During the study, the name of one } \\
\text { bronchitis bacterial. } \\
\text { AE, adverse event; SAEs, serious AEs }\end{array}$ & $\begin{array}{l}\text { of study treatment. } \\
\text { vestigator to be cau } \\
\text { rticipant's adverse }\end{array}$ & $\begin{array}{l}\text { related to the stuc } \\
\text { t reported was cha }\end{array}$ & $\begin{array}{l}\text { ug. } \\
\text { from bronchitis to }\end{array}$ \\
\hline
\end{tabular}

Patients receiving ESA therapy are exposed to EPO concentrations beyond physiological variations. Furthermore, patients with ESA hypo-responsiveness require higher ESA doses, likely increasing the risk of cardiovascular events [17-19]. In contrast, a 15-day multiple dose study in subjects undergoing hemodialysis showed that enarodustat increased $\mathrm{Hb}$ level together with physiological and dose-dependent increases in EPO concentrations without apparent accumulation after repeated doses [20]. In the present study, EPO concentrations also remained within a physiological range across enarodustat arms.

During the course of the study, 13 subjects had an AE of hypertension, all of which were considered subjects' underlying disease or worsening thereof. No AEs of hypertension were reported in subjects without complication of hypertension. No increases in mean systolic or diastolic blood pressure were observed during treatment in the safety population (data not shown).

In Period 1, enarodustat-treated subjects showed a tendency toward having lower hepcidin and ferritin and higher TIBC than those receiving the placebo. Although the mechanism of hepcidin suppression remains obscure, these results suggest that enarodustat may induce effective iron utilization. Functional iron deficiency is a target of concerns in anemic patients with CKD. In such patients, chronic inflammation may lead to increased hep- 
cidin production [21-23], inhibiting the uptake and mobilization of iron. Patients with CKD receiving ESAs are prone to iron deficiency because of increased demand for iron to support erythropoiesis; iron deficiency is the commonest cause of hypo-responsiveness to ESA therapy [24-26]. Given the changes in iron-related parameters observed in this study, enarodustat may support erythropoiesis by using available iron stored in the body. However, the comparative effects of enarodustat and ESAs on iron metabolism require further investigation.

Regarding safety, no apparent evidence of a dose-dependent increase in the incidence of AEs was observed.

Because HIF regulates VEGF expression, HIF stabilization may enhance it [27], thus contributing to angiogenesis-related diseases such as diabetic retinopathy and cancer [28]. As with other HIF-PH inhibitors, the present study showed no change in plasma VEGF after enarodustat treatment $[29,30]$. AEs considered attributable to enhanced VEGF expression should be profiled in Phase 3 studies on the long-term safety of enarodustat.

No active comparators were used in the study. However, a relationship between enarodustat dose and $\mathrm{Hb}$ response was found in the Period 1 in which placebo-controlled design was employed. In addition, $\mathrm{Hb}$ levels should be maintained within a target range during the maintenance therapy for renal anemia. Results obtained during the Period 2 revealed that $\mathrm{Hb}$ levels could be maintained within the target range when a dose of enarodustat was adjusted based on $\mathrm{Hb}$ levels. Thus, the findings in the study enable us to design the ongoing Phase 3 studies including comparative studies with an ESA.

In conclusion, enarodustat ameliorates renal anemia and maintains adequate $\mathrm{Hb}$ levels via dose adjustment in patients with CKD not on dialysis, and is well-tolerated. Effects on iron-related parameters suggest that enarodustat improves iron utilization. The efficacy and safety of enarodustat will be further examined in an ongoing Phase 3 studies including an ESA comparative study.

\section{Acknowledgments}

We would like to thank all of the physicians, nurses, and patients at the participating centers for their support. We acknowledge the editional support of ASCA Corporation (http://www.asca-co.com/english_site/) for proofreading a draft of this manuscript.

\section{Ethics Statement}

All patients provided written informed consent prior to participation. The study was registered with the Japan Pharmaceutical International Center (JapicCTI-152881) and was conducted in compliance with the ethical principles of the Declaration of Helsinki, the protocol, and the Guidelines for Good Clinical Practice of the Japanese Ministerial Ordinance and was approved by the Institutional Review Board of each participating study site.

\section{Disclosure Statement}

T.A., T.Y., and H.H. are consultants and received consulting fees from Japan Tobacco Inc. M.N. is a consultant and has received grants and personal fees from Japan Tobacco Inc. M.A., A.M., and R.K. are employees of and have stock or stock options in Japan Tobacco Inc.

\section{Funding Source}

Funding for this research was provided by Japan Tobacco Inc.

\section{Author Contributions}

All authors contributed to the final analysis and interpretation of data and had full access to the study data and analyses. All authors contributed to revising the article, providing intellectual content of clinical importance to the work described, and gave final approval for the version to be published.

\section{References}

1 Artunc F, Risler T: Serum erythropoietin concentrations and responses to anaemia in patients with or without chronic kidney disease. Nephrol Dial Transplant 2007;22:29002908.

2 Babitt JL, Lin HY: Mechanisms of anemia in CKD. J Am Soc Nephrol 2012;23:1631-1634.

3 Vos FE, Schollum JB, Coulter CV, Doyle TC, Duffull SB, Walker RJ: Red blood cell survival in long-term dialysis patients. Am J Kidney Dis 2011;58:591-598.
4 Scrutinio D, Passantino A, Santoro D, Catanzaro R: The cardiorenal anaemia syndrome in systolic heart failure: prevalence, clinical correlates, and long-term survival. Eur J Heart Fail 2011;13:61-67.

5 Silverberg DS, Wexler D, Blum M, Keren G, Sheps D, Leibovitch E, et al: The use of subcutaneous erythropoietin and intravenous iron for the treatment of the anemia of severe, resistant congestive heart failure improves cardiac and renal function and functional cardi- ac class, and markedly reduces hospitalizations. J Am Coll Cardiol 2000;35:1737-1744.

6 Silverberg D, Wexler D, Blum M, Wollman Y, Iaina A: The cardio-renal anaemia syndrome: does it exist? Nephrol Dial Transplant 2003; 18(suppl 8):viii7-viii12.

7 Pfeffer MA, Burdmann EA, Chen CY, Cooper ME, de Zeeuw D, Eckardt KU, et al: A trial of darbepoetin alfa in type 2 diabetes and chronic kidney disease. N Engl J Med 2009;361: 2019-2032. 
8 Singh AK, Szczech L, Tang KL, Barnhart H, Sapp S, Wolfson M, et al: Correction of anemia with epoetin alfa in chronic kidney disease. N Engl J Med 2006;355:2085-2098.

9 Szczech LA, Barnhart HX, Inrig JK, Reddan DN, Sapp S, Califf RM, et al: Secondary analysis of the CHOIR trial epoetin-alpha dose and achieved hemoglobin outcomes. Kidney Int 2008;74:791-798.

10 Drüeke TB, Locatelli F, Clyne N, Eckardt KU, Macdougall IC, Tsakiris D, et al: Normalization of hemoglobin level in patients with chronic kidney disease and anemia. N Engl J Med 2006;355:2071-2084.

11 Hirota K, Semenza GL: Regulation of hypoxia-inducible factor 1 by prolyl and asparaginyl hydroxylases. Biochem Biophys Res Commun 2005;338:610-616.

12 Haase VH: Hypoxic regulation of erythropoiesis and iron metabolism. Am J Physiol Renal Physiol 2010;299:F1-F13.

13 Wang GL, Jiang BH, Rue EA, Semenza GL: Hypoxia-inducible factor 1 is a basic-helixloop-helix-PAS heterodimer regulated by cellular O2 tension. Proc Natl Acad Sci U S A 1995;92:5510-5514.

14 Ke Q, Costa M: Hypoxia-inducible factor-1 (HIF-1). Mol Pharmacol 2006;70:14691480.

15 Peyssonnaux C, Zinkernagel AS, Schuepbach RA, Rankin E, Vaulont S, Haase VH, et al: Regulation of iron homeostasis by the hypoxia-inducible transcription factors (HIFs). J Clin Invest 2007;117:1926-1932.
16 Akizawa T, Hanaki K, Arai M: JTZ-951, An oral novel HIF-PHD inhibitor, elevates hemoglobin in Japanese anemic patients with chronic kidney disease not on dialysis. Nephrol Dial Transplant 2015;30(suppl 3):iii196.

17 McCullough PA, Barnhart HX, Inrig JK, Reddan D, Sapp S, Patel UD, et al: Cardiovascular toxicity of epoetin-alfa in patients with chronic kidney disease. Am J Nephrol 2013;37:549558.

18 Jelkmann W: The ESA scenario gets complex: from biosimilar epoetins to activin traps. Nephrol Dial Transplant 2015;30:553-559.

19 Fukuma S, Yamaguchi T, Hashimoto S, Nakai S, Iseki K, Tsubakihara Y, et al: Erythropoiesis-stimulating agent responsiveness and mortality in hemodialysis patients: results from a cohort study from the dialysis registry in Japan. Am J Kidney Dis 2012;59:108-116.

20 Pai S, Koretomo R, Tamaki S, Berg J, Marbury T, Galloway C, et al: JTZ-951, a novel HIF-PHD inhibitor, demonstrates increases in hemoglobin, iron mobilization, reproducible pharmacokinetics, and safety following once daily administration for 15 days in patients with anemia receiving hemodialysis. Nephrol Dial Transplant 2015;30(suppl 3):iii293-iii294.

21 Nicolas G, Chauvet C, Viatte L, Danan JL, Bigard X, Devaux I, et al: The gene encoding the iron regulatory peptide hepcidin is regulated by anemia, hypoxia, and inflammation. J Clin Invest 2002;110:1037-1044.

22 Malyszko J, Mysliwiec M: Hepcidin in anemia and inflammation in chronic kidney disease. Kidney Blood Press Res 2007;30: 15-30.

23 Ganz T: Molecular control of iron transport. J Am Soc Nephrol 2007;18:394-400.

24 Akizawa T, Okumura H, Alexandre AF, Fukushima A, Kiyabu G, Dorey J: Burden of anemia in chronic kidney disease patients in Japan: a literature review. Ther Apher Dial 2018;22:444-456.

25 Ganz T, Nemeth E: Iron balance and the role of hepcidin in chronic kidney disease. Semin Nephrol 2016;36:87-93.

26 Locatelli F, Fishbane S, Block GA, Macdougall IC: Targeting hypoxia-inducible factors for the treatment of anemia in chronic kidney disease patients. Am J Nephrol 2017;45:187-199.

27 Liu Y, Cox SR, Morita T, Kourembanas S: Hypoxia regulates vascular endothelial growth factor gene expression in endothelial cells. Identification of a $5^{\prime}$ enhancer. Circ Res 1995; 77:638-643.

28 Goel HL, Mercurio AM: VEGF targets the tumour cell. Nat Rev Cancer 2013;13:871-882.

29 Brigandi RA, Johnson B, Oei C, Westerman M, Olbina G, de Zoysa J, et al: A novel hypoxia-inducible factor-prolyl hydroxylase inhibitor (GSK1278863) for anemia in CKD: a 28day, phase $2 \mathrm{~A}$ randomized trial. Am J Kidney Dis 2016;67:861-871.

30 Martin ER, Smith MT, Maroni BJ, Zuraw QC, deGoma EM: Clinical trial of vadadustat in patients with anemia secondary to stage 3 or 4 chronic kidney disease. Am J Nephrol 2017; 45:380-388. 